\title{
TRA DOTTRINA GRAMMATICALE E ORTOGRAFIA LATINA: L'ALDINA DEL DE AETNA DI PIETRO BEMBO
}

\section{Giorgio di Maria}

Università degli studi di Palermo, 61, Piazza Marina, P90133, Palermo, Italia; giorgio.dimaria@unipa.it

Diacritical marks introduced by Pietro Bembo with the help of his editor, Aldus Manutius, in the first edition of his dialogue De Aetna form a complex and well-structured system. Although devised for practical purposes, so as to ensure a correct reading and phrasing of the Latin text, this system is clearly based on solid theoretical premises. The decisive factor for its introduction was the experience of editing Greek texts, which became the impulse as well as the basis for the system of diacritical signs. The influence of Latin grammarians (in particular, Servius and Priscianus), who had applied Greek accent theory to Latin language, also played an important part. Within a system essentially devoid of ambiguity, the three accents conveyed a variety of indications bearing on the correct pronunciation of the text, from basic principles to expressive nuances. Several editorial decisions involving phrase accents suggest that Bembo considered not only private reading, whether oral or mental (endophasic), but also public declamation.

Keywords: Pietro Bembo, Aldus Manutius, De Aetna, accents.

L'influenza che esercitò l'Aldina del De Aetna di Pietro Bembo, pubblicata a Venezia nel febbraio del $1496^{1}$, nella definizione del sistema paragrafematico del volgare italiano è stata riconosciuta da Arrigo Castellani in uno scritto che ha goduto della meritata risonanza $^{2}$. Lo studioso, esaminando anche le peculiarità di accenti riscontrate nell'incunabolo di tale opuscolo latino, le ha opportunamente ricollegate da una parte con lesperienza della tipografia di Aldo Manuzio, allora di recente apertura, la quale aveva inizialmente stampato quasi soltanto testi greci, e dall'altra con l'esperienza dell'Autore, reduce da un soggiorno biennale presso Costantino Lascaris a Messina, consacrato allo studio della grammatica e delle lettere greche. E nel nome del Lascaris il Manuzio ed il Bembo s'erano incontrati anche l'anno precedente, quando questi, di ritorno dalla Sicilia assieme ad Angelo Gabriel(e), gli consegnò per la nuova edizione la copia della grammatica greca che il maestro aveva affidata loro ${ }^{3}$.

Il Manuzio aveva bensì pubblicato un testo latino di notevole lunghezza (lo si sottolinea di rado perché esso, per la sua collocazione, può sfuggire all'attenzione di coloro che consultino indici e cataloghi): si tratta della ristampa della traduzione latina dichiaratamente letterale, di autore Giovanni Crastone ${ }^{4}$, inserita nelle pagine a fronte del greco proprio dentro lo spesso volume della grammatica, gli Erotemata, di Costantino Lascaris ${ }^{5}$

1 Bembo 1496.

2 Castellani 1995, 3-6, 11-12, 34-36: articolo rilevante anche per la fortuna del sistema accentuativo qui rappresentato.

3 Sicché lo stesso Aldo scrisse nelle premesse: "Ita vero emendatum manu ipsius Consta(n)tini libru(m) nobis dedere co(m)modo Petrus Bembus: \& Angelus Gabriel Patritii Veneti adeo nobiles: præstantiq(ue) ingenio Iuvenes": Lascaris 1495, a2 ${ }^{\mathrm{r}}$.

${ }^{4}$ La versione del Crastone era disponibile da almeno quindici anni, avendo già corredato il volume: Lascaris 1480; vd. Gualdo Rosa 1984, 579.

${ }^{5}$ Il frontespizio del volume, che funge anche da indice, tra l'altro si chiude con le parole: "Omnia suprascripta habent e regione interpretatione $(\mathrm{m})$ latina $(\mathrm{m})$ de verbo ad verbu $(\mathrm{m})$ ": Lascaris 1495 , al $\mathrm{r}$.

(C) St. Petersburg State University, 2016 
(nonché di alcune brevi aggiunte e appendici dello stesso Aldo); ma in quell'occasione trattavasi in massima parte di un testo 'di servizio', illeggibile per sé, finalizzato com'era unicamente a rendere spedita l'intelligenza del greco. Ivi il testo latino era stato pubblicato, come innumerevoli testi a stampa di fine Quattrocento, in stretta contiguità di forme, di stili (e anche di abbreviature, comunque piuttosto sporadiche) rispetto ai manoscritti coevi vergati nella scrittura umanistica.

Il De Aetna invece, rappresentando la prima opera letteraria in lingua latina che si sarebbe pubblicata per i tipi di Aldo Manuzio, meritava unattenzione particolare. In un'officina in cui il greco, con i suoi accenti e spiriti, era trattato con cura meticolosa, quella fu loccasione per interrogarsi se il latino non dovesse esser corredato di un sistema di accenti moderno. La risposta fu affermativa, tanto che con il De Aetna fu avviata una sperimentazione la quale, con adattamenti e semplificazioni, sarebbe stata portata avanti ivi per qualche decennio, ed avrebbe esercitato sullortografia del latino, nonché di alcune lingue romanze, l'influenza durevole mostrata dal Castellani, fissando qualche convenzione perdurante per quello fino agli albori dell'Ottocento, per queste tuttora vigente.

Tale sperimentazione, per la sistematicità e la duttilità con cui furono applicati i relativi segni (con diversi fenomeni di polisemia, comunque scevri di ambiguità, che ci sforzeremo di intendere e di commentare), si dimostrerà molto più organica ed ampia rispetto ad altri tentativi condotti in precedenza ${ }^{6}$ e soprattutto si rivelerà sostanzialmente indipendente dai tentativi dei precursori, in quanto assumeva direttamente dal greco, con intendimenti propri, gran parte dei mezzi utilizzati.

Il riesame che qui si propone avrà per oggetto gli accenti grafici (e altri segni consimili) nei rapporti fra le convenzioni ortografiche del greco e l'applicazione che se ne faceva al latino, cercando di enucleare da essa, per quanto possibile, qualche aspetto della cultura grammaticale che vi era sottesa. Non ci soffermeremo invece sui segni d'interpunzione, che tanto interessarono al Castellani, tematica diversa e scarsamente afferrabile al nostro sguardo retrospettivo, a motivo della grande varietà degli usi documentati nei manoscritti tra Medioevo e Rinascimento, nella quale facilmente si rinverrebbero i precedenti di ognuna delle diverse tendenze dellancor giovane tipografia latina.

I segni tipografici di nostro interesse che troviamo adoperati per il De Aetna sono quelli dellaccento acuto, circonflesso e grave, non sempre dotati, a parte il primo, della valenza di accenti, e quello di vocale lunga. La loro riconoscibilità era garantita dal nuovo stile grafico che era stato studiato per lopuscolo ${ }^{7}$ e s'inaugurava in quella circostanza, caratterizzato anche da vocali uniformemente basse e prive di significativi elementi sporgenti sopra la linea superiore delle minuscole (mentre i testi latini pubblicati l'anno precedente nel volume degli Erotemata presentavano vocali 'i' sistematicamente sormontate non da punti, ma da veri e propri apici obliqui indistinguibili da segni di accento acuto:

${ }^{6}$ Il Castellani cita (Loew 1980, 274-7) per esempi di codici di quell'area corredati di accenti (su monosillabi, su penultime lunghe di polisillabi, sulla sillaba che precede -que enclitico, su sillabe toniche); e poi parecchi codici singoli, in carolina come il cod. San Marco 387 del sec. XI (accenti soltanto acuti: su monosillabi, per indicazione della sillaba tonica, distinzione di omografi); e il San Marco 615 del sec. XI-XII, di pari caratteristiche; il Sess. 38 del sec. VIII (fitta presenza di accenti in un codice altomedievale, vergato in scrittura precarolina di Nonantola); usi che ritiene sostanzialmente tramontati entro fine XIII sec. Rileva poi in manoscritti vergati da Niccolò Niccoli un limitato, ma sistematico uso dell'accento. Vedasi: Castellani 1995, soprattutto $12-24$.

7 Si adoperavano notoriamente nuovi caratteri mobili disegnati all'uopo da Francesco Griffo da Bologna: Mardersteig 1988; Tinti 2002. 
siffatto stile tipografico sarebbe stato incompatibile con il sistema di accenti che s'introduceva nel De Aetna).

Consideriamo ora partitamente i segni utilizzati e le funzioni che assumono.

\section{Segno di accento acuto / circonflesso}

L'accento acuto ${ }^{8}$ indica la sede dell'ictus e ricorre diffusamente a scopo cautelativo, laddove si paventa erronea lettura, e sistematicamente come accento d'enclisi; ve ne sono inoltre alcune poche occorrenze (che discuteremo successivamente) a indicare l'accento sintagmatico o l'accento di frase.

Laccento circonflesso sulla penultima sillaba è parimenti adoperato a segnare la sede dell'ictus, purché questa sia lunga e l'ultima sia breve, nel rispetto della regola che vige in greco. Pertanto esso è adoperato raramente, siccome rari sono i luoghi in cui fu ritenuto opportuno segnare l'accento della penultima (astrazion facendo dall'accento d'enclisi che, posto prima dell'enclitica, sull'esempio del greco è sempre acuto prescindendo dalle quantità). Ove nella parola l'ultima sillaba in corso di flessione divenisse lunga, il circonflesso della penultima si muterebbe in acuto: ambîtus - ambitu (rispettivamente nominativo [-tŭs] e ablativo [-t $\bar{u}]$ del singolare), conformemente al modello di $\dot{\eta} \mu$ oṽ $\sigma \alpha-\tau \tilde{\eta} \varsigma$ $\mu o v ́ \sigma \eta \varsigma^{9}$. L'accento circonflesso in latino era stato teorizzato prevalentemente in età tardoantica, soprattutto da Servio In Donati artem maiorem (Serv. GL IV, 426.6-427.35) e da Prisciano nelle Institutiones (Prisc. GL II, 51.22-53.6).

Inoltre, il segno di accento circonflesso, che sulla penultima sillaba indica sia accento sia quantità vocalica di lunga, può essere adoperato sull'ultima sillaba, dove si esclude che abbia valore di accento, come mero indicatore di quantità (lunga).

Del segno di accento grave, adoperato, come modernamente in greco, soltanto sull'ultima sillaba, non mai a indicare la sede dell'ictus, ma ad escluderla, o a segnare mera quantità di lunga, tratteremo in prosieguo.

\section{Ortoepia e disambiguazione}

Gli accenti cautelativi, di cui si diceva, compaiono nei polisillabi ${ }^{10}$ laddove si desideri o prevenire un'erronea lettura (come nei composti accentati sul preverbio, in cui si paventa che il lettore continui ad usare l'accento del secondo elemento), o raccomandarne una fra due lecite (è il caso della positio debilis), o ancora per disambiguazione, nei casi in cui sono possibili due letture con diverso valore morfosemantico.

In tale funzione di presidio per la corretta lettura (prescindendo dal caso dell'enclisi) troviamo presenti nel De Aetna gli accenti grafici, nonché un moderno segno di quantità, su: $(\mathrm{B} 6 \mathrm{~V} 5)$

ita(que) $\left(\mathrm{A} 1^{\mathrm{v}} 1, \mathrm{~A} 2^{\mathrm{r}} 15, \mathrm{~A}^{\mathrm{v}} 1, \mathrm{~B} 7^{\mathrm{r}} 8, \mathrm{~B} 7^{\mathrm{v}} 17, \mathrm{C}^{\mathrm{v}} 7, \mathrm{C}^{\mathrm{v}} 9, \mathrm{C}^{\mathrm{v}} 13, \mathrm{D} 5^{\mathrm{v}} 19\right) \neq i$ táq(ue)

8 Ometteremo frequentemente la componente aggettivale di 'laccento grafico', nella misura in cui risultino abbastanza evidenti e facili da discernere i casi in cui ci riferiamo al segno della parola scritta.

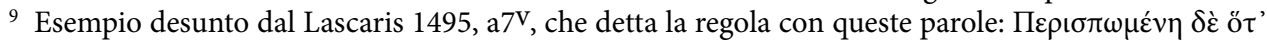

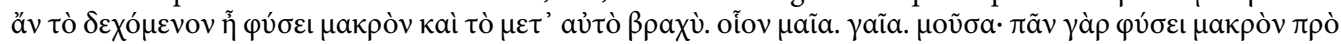

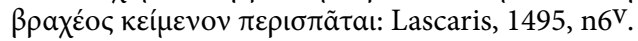

10 Intendiamo: parole di tre o più sillabe. 


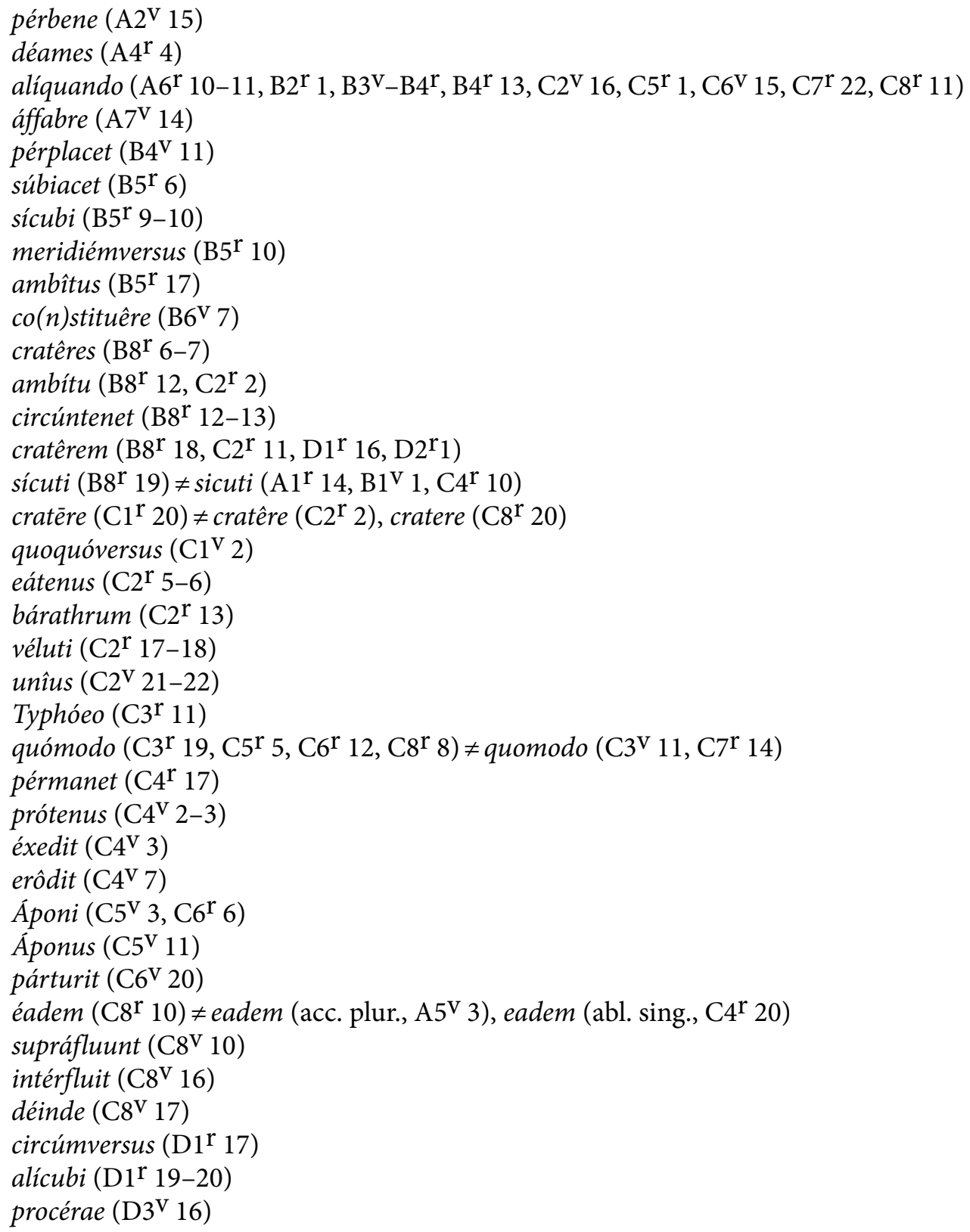

Fra questi termini, nei quali il Bembo ritenne opportuno fornire l'ausilio di un accento grafico ${ }^{11}$, riguardiamo brevemente quelli che possono presentare pur modeste difficoltà

${ }^{11}$ Quanto alla posizione dell'accento, il segno grafico è collocato precisamente sulla vocale interessata se minuscola; nel caso delle maiuscole, si presentò lesigenza tecnica di qualche adattamento: Íta(que) presenta tre volte l'iniziale maiuscola, nel qual caso il segno grafico dell'accento acuto, per tipografiche esigenze di spazio, è spostato in avanti sopra ' $\mathrm{t}$ ' (A $1^{\mathrm{V}} 1, \mathrm{~A} 2^{\mathrm{r}} 15, \mathrm{D} 5^{\mathrm{V}}$ 19); così Áponus (anche al genitivo Áponi) presenta nelle sue tre occorrenze l'iniziale maiuscola ed il segno grafico dell'accento acuto spostato in avanti sopra la lettera 'p'. 
prosodiche - considerando i necessari riferimenti alla dottrina grammaticale - od esigenze di disambiguazione.

Scrivendo ítaque si ricordava al lettore che l'elemento finale, integratosi in tale formazione già nei primordi del latino, non doveva esercitare attualmente gli effetti della congiunzione enclitica - que, la quale avrebbe implicato accento (anche grafico, nelle convenzioni del De Aetna) ${ }^{12}$ sulla penultima sillaba dell'unità accentuale costituentesi (e itáq(ue) farà la sua comparsa, problematica, al luogo segnato $)^{13}$.

Con áffabre e bárathrum si mirava anche e soprattutto a dissipar la perplessità ingenerata dalla cosiddetta positio debilis, che è data da doppia consonante, occlusiva seguita da liquida, al confine tra penultima e ultima sillaba dietro vocale breve per natura ${ }^{14}$, e la preferenza del Bembo per l'accento sulla terz'ultima sillaba collima con la prassi attuale, di confinare accentazioni sulla penultima alla poesia, sotto conforme evidenza prosodica.

In ambîtus, ambitu ${ }^{15}$ si nota che il Bembo spinge la propria raffinata ricerca di esattezza al punto di trattare il sostantivo ambĭtus alla stregua del supino e del participio perfetto di ambeo: ambìtum, ambìtus; a loro volta tali forme per quantità si oppongono a quelle di altri verbi composti a secondo elemento eo, quali transeo: transĭtum, transĭtus; adeo: adĭtum, adĭtus, rispetto alle quali i sostantivi derivati (transĭtus, adĭtus) presentano quantità di penultima sillaba ed accenti conformi; onde, forse, il convincimento che anche nel sostantivo ambitus la penultima sillaba fosse lunga come nelle forme verbali etimologicamente imparentate ${ }^{16}$.

In co(n)stituêre, ovviamente, il segno di accento esplicita il perfetto indicativo, in opposizione all'infinito constituĕre.

Se unĭus giunge a prevalere nella poesia esametrica ${ }^{17}$, i curatori del De Aetna, indicando con unîus la scansione a penultima lunga, anche in questo caso rivendicano alla prosa le sue prerogative.

In Typhóeo l'accento vuol mostrare anzitutto che 'o' interno suona col proprio timbro, lungi dal costituire dittongo e monottongarsi nel suono 'e’ con la successiva vocale (ché i poeti latini antichi verosimilmente scandivano le due vocali in iato, seguendo con la solita fedeltà il modello offerto dal greco) ${ }^{18}$; cionondimeno l'inosservanza dell'uso

12 Sistematicamente segnato per tutto il testo in caso di enclisi attuale di -que, vd. infra.

${ }^{13}$ Non differisce infatti sensibilmente per significato dagli altri. Esistette tuttavia nel latino antico un itaque equivalente ad un vero e proprio et ita, il quale è da pronunziar proprio itáque, ad es. nella formula del testamentum per aes et libram: HAEC UT IN HIS TABULIS CERISQUE SCRIPTA SUNT, ITA DO, ITA LEGO, ITA TESTOR ITAQUe vos, QUiRITES, TESTIMONIUM MIHI PERHIBETOTE (Gaius Inst. II, 104) Nondimeno nel passo del De Aetna sembra doversi ravvisare un mero errore tipografico, e forse non è casuale il fatto che dalla copia della Biblioteca Nazionale Centrale di Firenze (Ald. 1.1.40) il segno sia stato accuratamente abraso: potrebbe essere anche questo un frutto di una ben precisa attività di emendazione di cui si rinvengono le tracce, diffusamente, nelle copie del De Aetna sparse per le biblioteche di nazioni diverse, svolta nel tempo verisimilmente proprio nellofficina di Aldo Manuzio prima del loro rilascio commerciale; vd. Bühler 1951.

${ }^{14}$ Il Quicherat affianca i lemmi bărăthrŭm e bărāthrŭm e mostra la frequenza della scansione a penultima lunga nei testi poetici: tra i versi citati Sil. 9, 467: "Qui, se postquam Aetnae mersit candente barathro" (-räth-), che verte sull'Etna: Quicherat 1846, 139. Affabre non è d'uso poetico.

${ }^{15}$ Correttamente differenziandosi, almeno ai fini dell'alternanza circonflesso / acuto sulla penultima sillaba, il nom. sing. ambitus (uscente in -tŭs) e l’abl. sing. ambitu (in -tū).

16 Vd., ad vv. Quicherat 1846.

17 Vd. Quicherat 1846, 1268-1269.

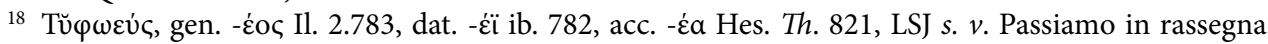
solo gli esempi della poesia augustea, con le scansioni che un sistema uniforme accredita (mentre le singole occorrenze non forniscono necessariamente indizi univoci): Tyр̆hōe $\widehat{u s}$ (Verg. Aen. 8 298), Ty̆phōe $\widehat{a}$ (Verg. 
classico e la prassi poetica seriore davano fin dalla Commedia di Dante come esito volgare Tifeo ${ }^{19}$.

Nell'indicativo pérmanet (del composto di măneo) concorre oltretutto l'esigenza della distinzione dal congiuntivo permānet (del composto di māno).

In éxedit vedesi differenziato il presente $(-x \breve{e}-)$ dal perfetto $(-x \bar{e}-)$.

Erôdit con accento circonflesso, confirmatorio della quantità della penultima $(-r \bar{o}-)$, si distingue vieppiù da erŭdit.

In Áponi e Áponus il relativo segno grafico manifesta unaccentazione prossima al volgare Ábano, e coerente con l'etimologia sostenuta dal Bembo, il quale rappresenta le relative terme come il luogo ä́rovos, in cui le cure si praticano anzitutto in modo indolore: "s in e ullo a egri dolore, qua de causa Graeco verbo Áponus nomen invenit [...]" $\left(\mathrm{C} 5^{\mathrm{V}} 10-12\right)^{20}$.

Con la grafia éadem fu ritenuto non superfluo, nel luogo segnato, manifestare che si trattava di un caso retto del neutro plurale, mentre analoga sollecitudine non è riscontrabile negli altri passi.

In déinde l'accento sulla prima vocale scongiurava anche il rischio di una pronunzia come trisillabo (non rispondente alla più raccomandabile prosodia antica) ${ }^{21}$, che, se si fosse accentuata la seconda, sarebbe scaturita immediatamente dalle consuetudini fonatorie degli Italiani.

Procérae si teneva ben distinto da procĕres, a scongiurare impropria lettura analogica.

Crater, nei due casi qui adoperati della sua flessione (cratêrem, cratēre ${ }^{22}$, cratêre), si distingueva dal tipo di ansĕr -ěris, lasĕr -е̌ris, tubĕr -е̌ris, non potendosi tuttavia escludere che l'identità del termine come prestito dal greco abbia invogliato maggiormente a corredarlo in modo sistematico di ausili alla lettura (per quanto altri prestiti, di parimenti manifesta origine greca, non ne siano corredati).

Accenti come alíquando, meridiémversus, quoquóversus, circúmversus ${ }^{23}$ mostrano il prevalere della dottrina grammaticale sulla legge della penultima: si vuol certo insistere

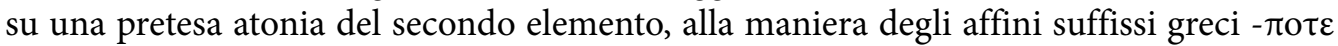
e $-\delta \varepsilon /-\theta \varepsilon v$, annoverati fra le enclitiche, che determinano accento (peraltro oggetto di discussione fra gli stessi grammatici antichi) per lo più sull'ultima sillaba dell'elemento che si unisce ad essi. Cf. in particolare, per alíquando, $\pi \omega \dot{\pi} \tau \tau \varepsilon$ e gli altri composti, frequentissi$\mathrm{mi}$, a secondo elemento $-\pi \mathrm{o} \tau \varepsilon^{24}$, e per quoquóversus, circúmversus i loro omologi rispettivi

Georg. I 279), Ty̆phōěă (Verg. Aen. 1, 665; al confine fra $4^{\circ}$ e $5^{\circ}$ piede, onde il Ribbeck e gli altri maggiori editori evinsero la necessità di scrivere Typhoëa).

19 Cf. Dante, Paradiso VIII 70; Petrarca? Triumphus pudicitiae 113.

${ }^{20}$ Mentre invenit non è stato corredato di un accento, a distinguere il perfetto indicativo dal presente.

21 Vd., al lemma Děīn, Děīncēps, Děīndě et mieux Deīn, Deīncēps, Deīndě: Quicherat 1846, 329.

22 Con segno di quantità, l'unico presente in tutto il libro, orizzontale e non inclinato, del tutto coincidente con l'uso moderno. Lalternanza di grafie quali cratēre, cratêre era disomogeneità tollerabile, specie se si consideri che i segni di quantità erano annoverati tra gli accentus: Manutius 1508, aa8 r.

${ }^{23}$ Se circumversus avverbio fu preferito ad evitare circumcirca in quanto postclassico, il rimedio sembra peggiore del male: escludendo ovviamente voci verbali di circumverto, non l'abbiamo trovato attestato prima di Rabano Mauro [De vita B. Mariae Magdalenae et Marthae // PL 112, 1493B].

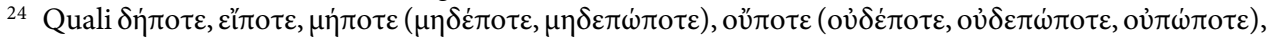
$\pi \omega \dot{\pi} \pi \tau \varepsilon, \tau i \pi o \tau \varepsilon$. Non si può sottovalutare che Aldo Manuzio pochi anni dopo spiegherà il fatto a modo proprio citando lo stesso alíquando in un elenco di termini in cui l'accento, irregolare, esprimerebbe l'unità della parola altrimenti soggetta ad esser letta come due membri distinti: "Non servatur autem accentus modis septem: [...] Ambiguitate, ad evitandam amphibologia(m), ut intereáloci. alíqua(n)do, síquando, néquando, Déinde, éxinde, périnde, próinde, súbinde, dúntaxat, quæ antepenultimam acuunt ne duæ partes 


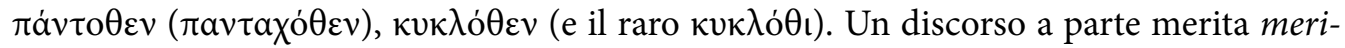
diémversus, in cui si deve preliminarmente ravvisare una precisa scelta del Bembo, per il quale il rapporto sostantivo-suffisso diviene esclusivo, soppressa, come in antichi scrittori tecnici, la preposizione $a d$ che i letterati sentivano come costitutiva dell'espressione. Cf. ad meridiem versus (Varro Rust. I, 2, 4; Liv. I, 18, 7; Curt. V, 4, 7; Mela I, 81; Plin. Nat. V, 43), che troverebbe bensì il suo corrispondente greco, nel semplice $\pi \rho \grave{\varsigma} \varsigma \mu \varepsilon \sigma \alpha \mu \beta \rho i \eta \nu$ (Hdt. I,

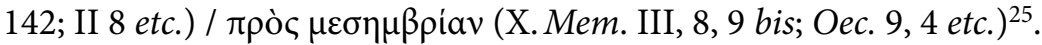

Interessante è esaminare le restanti parole dell'elenco preposto, nelle quali l'accento fu segnato a fini ortoepici pur in assenza di intrinseche difficoltà obiettive:

pérbene, déames, pérplacet, súbiacet, sícubi, circúntenet, sícuti, eátenus, véluti, quómodo, pérmanet, prótenus, párturit, supráfluunt, intérfluit, alícubi.

Tale breve elenco dà la misura di certa sfiducia del Bembo e del Manuzio nel lettore contemporaneo, esposto per imperizia al rischio di infrangere le leggi ortoepiche al momento di leggere quei termini, composti e non ${ }^{26}$.

Ma forse il quadro non è semplice come appare al filologo di oggi, dalla solida formazione linguistica (come si auspica): erano quelli ancora anni di assestamento, in cui il testo a stampa, esistente da alcuni decenni, al riguardo di non pochi fra quei termini (e di molti altri, estranei al De Aetna), cominciava finalmente a stabilizzarsi e a chiarire con la grafica nitida e le spaziature uniformi del contesto che circun-tenet, sic-uti, ea-tenus, vel-uti, quo-modo, supra-fluunt, inter-fluit, lungi dallessere separati, in quanto costituiti da elementi saldamente entrati in composizione andavano scritti senza soluzione di continuità; i manoscritti invece, con tutte le esitazioni degli scribi, sorte nel momento in cui la scriptio continua si era progressivamente sciolta nella separatio verborum e non mai cessate da allora, continuavano ad alimentare incertezze sull'unità di quelli, che davano adito a letture esitanti. Pertanto è lecito ritenere che in circúntenet, sícuti, eátenus, véluti, quómodo, supráfluunt, intérfluit (se non in altri ancora dellelenco quivi premesso) il segno grafico, pur ridondante, assuma comunque la funzione di rendere vieppiù evidente l'unità del composto prescrivendo l'accento che ne consegue.

Del resto Aldo Manuzio, anche dopo la stampa del De Aetna, continuerà ad adoperarsi in favore di una lettura corretta dei verbi composti con preverbio e fornirà al riguardo norme chiare e precise nella sua grammatica ${ }^{27}$.

e(ss)e putentur"; ove intendiamo: "Non servatur autem accentus modis septem" come non si osserva la regola generale dell'accento in sette casi: Manutius 1508 , aa7 $\mathrm{V}$.

${ }_{25}$ Il passo più antico che presenti il costrutto privo di $a d$ sembra trovarsi in Solino, a motivo della lingua tecnica, della complessità delle informazioni e dei complementi di luogo forniti nel contesto: "Ergo Italia, in qua Latium antiquum antea a Tiberis ostiis ad usque Lirim amnem pertinebat, universa consurgit a iugis Alpium, porrecta ad Reginum verticem et litora Bruttiorum, quo in maria meridiem versus protenditur" (Sol. II, 19); segue Orosio, paragonabile per tecnicismi e dovizia di informazioni topografiche: "[...] MARE CASPIVM sub Aquilonis plaga ab oceano oritur [...]. inde meridiem versus per longas angustias tenditur, donec per magna spatia dilatatum Caucasi montis radicibus terminetur" (Oros. Hist. I, 2, 48). Non mancano in entrambi gli autori costrutti analoghi riferiti ad altri punti cardinali, come Orientem versus.

26 Ad es.párturit, accentato pur non trattandosi di un verbo composto nébisognoso di disambiguazione, dà motivo di ritenere che esistessero già nel Rinascimento lettori pronti, come lo studente universitario di oggi, a lasciarsi sfuggir di bocca dopo partúrio anche monstra fonici quali partúris e partúrit.

27 Manutius 1508, aa6 $\mathrm{V}_{-}$aa7 $\mathrm{r}$. 
Resta, difficile da analizzare, alíqua, un unicum, fra l'altro in versuum discrimine (alí|qua) mentre il termine compare altre due volte, in nominativo e in ablativo, scritto aliqua senza accenti, come ne son prive tutte le forme di aliquis/aliqui. È lettura talmente indifendibile da far supporre un errore tipografico favorito da fine di rigo, quasi loperatore avesse creduto per un attimo di star componendo l'avverbio non infrequente alíquando.

\section{Accento d'enclisi}

Laccento d'enclisi è segnato graficamente in modo sistematico e non mai omesso, certo sotto l'influenza del greco, il cui sistema coerente e rigoroso, adoperato da secoli nei manoscritti bizantini, era allora fedelmente ripreso nella stampa. Dalla prassi di tale lingua si desumevano l'assiduità e l'attenzione nel segnarlo, mentre la molteplice casistica del greco conosceva una drastica semplificazione dinanzi all'uniformità del latino: accento acuto sull'ultima sillaba, invariabilmente, della parola alla quale è affissa l'enclitica.

Nella pratica, si noti che -que comporta accento d'enclisi, acuto, sulla sillaba che lo precede, anche quando si affigga a un monosillabo, senza eccezione, tutte le volte che sia vera congiunzione enclitica. Viceversa non compare tale accento grafico tutte le volte che -que si è storicamente integrato nel termine sì da non essere più sentito come congiunzione.

Sarebbe lungo elencare gli esempi dell'uno e dell'altro caso: basti per il secondo specificare che atque, denique, quandoque, circunquaque e usquequaque, plerique, quicunque, quisque e quoque, uterque (frequentemente abbreviati) non sono stati corredati d'accento grafico in alcuna delle loro occorrenze. Così neque, se non fosse per una sola delle sue ventinove occorrenze: "Q(uod) reip(ublicae) causa non commoveri neq(ue) vellem, si possem, néq(ue) possem, si velim" (A $\left.4^{\mathrm{r}} 13-15\right)$, laddove il secondo, néque, in apertura del suo brevissimo inciso, si differenzia forse dal primo per un marcato accento di frase.

Se viceversa itaque compare sistematicamente con l'accento grafico sulla sillaba iniziale (tanto che l'abbiamo incluso nell'elenco di sopra, fra i termini suscettibili di disambiguazione), significa che fu tenuta presente l'eventualità di un itáque che valesse et ita, e di tale accento grafico si reperisce un solo esempio del De Aetna, pur sprovvisto di quella precisa valenza ${ }^{28}$. Ancora, si rinvengono quáque, nel solo passo in cui vale et qua $\left(\mathrm{D} 1^{\mathrm{r}} 21\right)$, e quámq (ue), $q\left(\right.$ uám $q(\text { ue })^{29}$, nei soli passi in cui valgono et quam (rispettivamente $\mathrm{C}^{\mathrm{r}} 3$, $\mathrm{D} 2^{\mathrm{r}} 3$ ). L'accuratezza con cui sono state seguite queste norme in un incunabolo è tale da destar ammirazione, se si consideri la frequenza dellelemento -que, che ricorre più volte quasi in ogni pagina; essa è sicura conferma dell'utilizzo di maestranze colte, avvezze a trattare il greco.

La particella -ce compare solo nella forma tronca $-c$ e non comporta, né in ragione dell'enclisi né in ragione del troncamento, accento grafico sulla sillaba che la precede; visto il trattamento unitario, non mette conto distinguere istoc (abl. di iste: "istoc aestu", $\mathrm{A} 5^{\mathrm{r}}$ 16), in cui sarebbe da ravvisare descrittivamente vera enclisi perché l'elemento $-c$, non necessario, apporta la propria significazione epidittica o intensiva, da istuc, adhuc,

28 Vd. sopra, n. 13.

${ }^{29} q($ uám $) q($ ue $)$ reca l'accento grafico tra i due $q$, spostato in avanti rispetto al primo 'q' perché esso è già sormontato da segno di abbreviazione. 
illic (avverbi di luogo e di tempo), di cui la particella storicamente è diventata elemento costitutivo sicché l’autore, riferendosi alla morfologia del latino classico, non ha facoltà di ometterla. Se nemmeno il troncamento ha indotto a segnare accento sull'ultima, considerato il grado di elaborazione del testo e la dovizia di ausili offerti al lettore, vien da pensare che l'accento si pronunziasse indiscusso sullattuale penultima.

Lenclitica -ne comporta accento d’enclisi in méne $\left(\mathrm{B} 3^{\mathrm{V}} 19\right)$, laddove vi è propriamente enclitica; e sembra che sia stato trattato a guisa di composto con enclitica - il che non è del tutto irragionevole né incompatibile con il senso - anche nónne, scritto così nelle sue due occorrenze $\left(\mathrm{C}^{\mathrm{V}} 1, \mathrm{C}^{\mathrm{V}} 16\right)$; altri nondimeno potrebbe ipotizzarvi accento di frase anziché d'enclisi, segnato graficamente a prescrivere pronunzia marcata perché in tale sede ha inizio il tono interrogativo che dovrà connotare tutta la frase. In ultimo, anche in ain' ( $\left.\mathrm{A} 8^{\mathrm{V}} 3\right)$ è presente un segno, ma solo di troncamento, l'apostrofo. Se ne ricava un quadro coerente, a conferma che le enclitiche tronche in latino non comportano per il Bembo accento d'enclisi.

L’enclitica -ve comporta accento d'enclisi nella sua unica occorrenza: vetustásve (B1 ${ }^{\mathrm{V}}$ 6-7). La congiunzione sive, correttamente, è sprovvista di accento grafico.

In ultimo, -met, nella prassi di Bembo-Manuzio, non comporta accento d'enclisi, come osservabile in sibimet $\left(\mathrm{C} 4^{\mathrm{r}} 14\right)$ e in semet $\left(\mathrm{C}^{\mathrm{V}} 19-20\right)$.

Viceversa in mécum $\left(\mathrm{B}^{\mathrm{r}} 21\right)$ sembra di ravvisare qualcosa di analogo ad un accento d'enclisi, se non un segno confirmatorio della grafia unita, senza soluzione di continuità, del pronome con la preposizione in anastrofe. Tuttavia l'esempio è particolarmente incerto e poco si presta a produrne deduzioni, visto che altrove si leggono nobis cum $\left(\mathrm{B}^{\mathrm{V}}{ }^{5}\right)$ con spazio interposto e tecum $\left(\mathrm{B} 2 \mathrm{~V} 16, \mathrm{D}^{\mathrm{V}} 21\right)$ sprovvisto di accento grafico.

In conclusione, le enclitiche che nel De Aetna comportano accento grafico d'enclisi sono, in ordine di frequenza decrescente, $-q u e,-n e,-v e^{30}$.

Se si tiene presente che la misura di tutti gli altri provvedimenti qui adottati in materia di accento fu comunque dettata da sobrietà ed economia, la costanza nell'apposizione dell'accento d'enclisi potrebbe far supporre che esso fosse materia di particolare incertezza, almeno per una parte del pubblico al quale si indirizzava il De Aetna.

\section{Accento d'anastrofe}

Si osservi anche il caso, unico e interessante, della preposizione sine, scritta di regola senza accento $\left(\mathrm{C} 3^{\mathrm{v}} 13, \mathrm{C}^{\mathrm{r}} 18, \mathrm{C}^{\mathrm{v}} 10, \mathrm{C}^{\mathrm{r}} 1, \mathrm{C}^{\mathrm{r}} 16\right)$, la quale lo riceve invece nell'unico passo in cui è posposta, "qua síne [sc. ea pars] ad ignes ipsos perveniri non potest [...]" $(\mathrm{B} 7 \mathrm{~V}$ 11-12). Anche in questo caso funge da modello il greco, in quanto si vuole che vi si

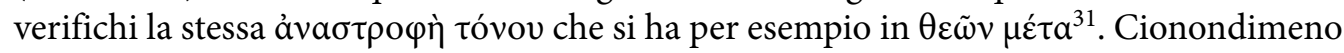

${ }^{30}$ Non diverso l'elenco delle enclitiche aventi influenza sullaccento che Aldo Manuzio fornirà nel 1506, nella sua grammatica latina così ricca di indicazioni ortotipiche: "Necessitate non servatur accentus,

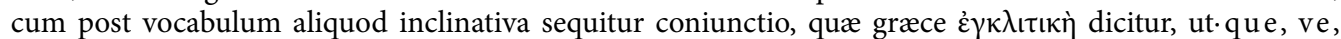

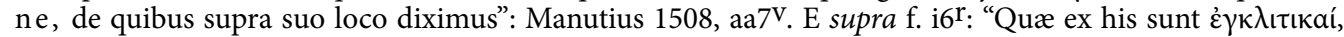
idest encliticæ, hoc e(st) inclinativæ? que. ve. ne. Quare dicuntur $\dot{\varepsilon} \gamma \kappa \lambda \iota \tau \iota \kappa \alpha i ́ ?$ quia inclina(n)t accentum præcedentis syllabæ ad se...”

31 Alcuni anni più tardi Aldo Manuzio formalizzerà una regola chiaramente ispirata al greco, ma di una portata che ne trascende nettamente i limiti, anche nellesempio latino addotto: "Transpositione [sc. non servatur accentus], cum de suo loco dictio transmutatur, ut sunt præpositiones, quæ præpositæ gravantur, ut dè quo genere mortis difficile dictu est. Postpositæ autem, quia mutant locum, mutant etiam accentum, 
in latino l'accento è da ritenersi più che altro un puro segno grafico, essendo la sillaba già normale sede di accento, mentre in greco si verifica un reale ed effettivo spostamento dell'accento sintagmatico, non potendosi comunque escludere una componente d'enfasi qual è quella che si ha in grado maggiore nell'accento di frase. Una pronunzia accurata serve anche a differenziare più nettamente qua sine da quasi ne, e questo passo, come tutti i passi che involvono l'accento sintagmatico o l'accento di frase, conferma che nell'umanista Bembo il latino era esente da quel profondo iato fra parola scritta e parola pronunziata / recitata, iato che spesso alberga nella mente del latinista di oggi, avvezzo a tenere sotto gli occhi il testo più che ad ascoltarlo.

Alla luce di qua síne si è forse in grado di comprendere la scrittura antè nos come ulteriore, sofisticata forma di disambiguazione. "Is enim paucos antè nos dies per summam tranquillitatem totum verticem perlustrarat" $\left(\mathrm{C}^{\mathrm{V}} 13-15\right)$. Nel passo irto di accusativi, probabilmente si vuol prevenire un travisamento che faccia di ante la preposizione reggente l'accusativo paucos dies, distribuito dinanzi e dietro di essa, anziché, comè mente dell'autore, il pronome personale, nos parimenti in accusativo, che la segue immediatamente. Nel lettore può insinuarsi il dubbio, tanto più che paucos ante dies è espressione corretta e frequente: ma con i segni di accento che gli umanisti Bembo e Manuzio miravano ad introdurre, verosimilmente, le espressioni si sarebbero differenziate a questo modo: paucos ánte dies, prendendo ante posposto, pur soltanto all'aggettivo della sua reggenza, l'accento acuto sulla penultima sillaba; paucos antè nos dies, in cui l'accento grave esplicita che, non verificandosi il caso precedente, il reggimento della preposizione è da ricercarsi in quel nos che, normalmente, la segue.

\section{Accento sintagmatico, accento di frase}

Due tra i parecchi, disparati ausili che il Bembo offre vertono sulla lettura del quam. I mezzi adoperati manifestano la sua sollecitudine di evitare che il lettore si soffermi troppo sulla congiunzione, che deve mantenersi atona; tale può essere il caso, secondo l'Autore, laddove essa apra la proposizione o segua ante (pur con interposta spaziatura). Non è chi non veda lanalogia con il greco, in cui rispettivamente $\dot{\omega} \varsigma$ è congiunzione proclitica ed

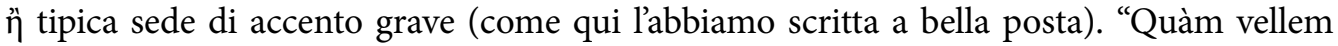
ut $[\ldots]$ possem ego $[\ldots]$ ” $\left(\mathrm{A} 8^{\mathrm{V}} 11\right)^{32}$. La proposizione è esclamativa, e il Bembo significa con l'uso dell'accento grave che il primo sensibile accento intensivo dev'essere collocato sul successivo vellem, a sottolineare il concetto di volontà espresso dal parlante con inten-

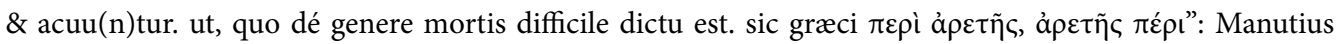
1508 , aa7 V Vi si noterà che l'esempio latino è di preposizione monosillabica, in cui pertanto non è possibile baritonesi, che diviene sede di accento acuto per quanto dopo la trasposizione continui ad esser preposta al sostantivo cui si riferisce. Nel De Aetna non vi sono esempi di una siffatta radicalizzazione del modello greco. Riguardo alla norma greca si ricordi infatti che notoriamente, "wenn die Präposition zwischen einem Substantive und einem Adjektive oder zwischen einem Eigennamen und einem Gemeinnamen steht, so tritt die Anastrophe ein, wenn das Substantiv oder der Eigenname der Präposition vorangeht, und das Adjektiv oder Gemeinname ihr nachfolgt, indem hier tatsächlich eine Nachstellung stattfindet, [...] geschieht aber nicht, wenn das Adjektiv oder der Gemeinname vorangeht, indem dann die Präposition vor dem wichtigeren Wort steht": Kühner 1890, I, 1, 334.

${ }^{32}$ Qui per quam non sono adoperate abbreviature (altrove sistematiche), anche in ragione dell'iniziale maiuscola. 
sa partecipazione affettiva ${ }^{33}$. Qui, e negli exempla che seguiranno, la valenza dell'accento grave non differisce da quella che riscontriamo nei papiri greci, soprattutto di età romana, quando in mancanza di una sistematica indicazione della vocale sede di accento (acuto o circonflesso) si connotavano qua e là con l'accento grave le sillabe che non erano sede di quegli accenti. Si potrebbe pensare che in età rinascimentale dovesse trattarsi di un uso obsoleto e ormai dimenticato; però i maestri e in generale gli eruditi non potevano non averne memoria, se non altro grazie alle stesse denominazioni: se ci riferiamo all'ultima sillaba,

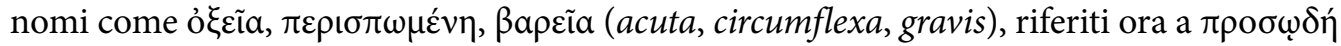
ora a $\sigma v \lambda \lambda \alpha \beta \eta$, richiamavano continuamente al parlante colto i rispettivi valori dei segni, corrispondenti a modi sensibilmente diversi, almeno in antico, di profferire la sillaba che ne era sede.

Lo stesso Costantino Lascaris alle lezioni non poteva trascurare tale aspetto, espresso chiaramente nella sua grammatica che s'era ristampata a Venezia per i tipi di Aldo Manuzio $^{34}$.

“[...] sed opus est ánte, quam illò veniam; ut aliqua [...]" $\left(\mathrm{A}^{\mathrm{r}}{ }^{5-6}\right)$

"[...] iam inde ánte, $\mathrm{q}(\mathrm{uam})$ tu es natus [...]" $\left(\mathrm{B} 1^{\mathrm{r}} 10\right)^{35}$

"[...] ánte, q(uam) istuc accessisses [...]" (B7 $\left.{ }^{\mathrm{r}} 21-22\right)$

Se all'interno della congiunzione composta ánte quam il Bembo desidera uno stacco, brevissimo ma certo non nullo (probabilmente più una precisa articolazione dei membri che una pausa), e la prescrive sia con la scriptio distincta sia con l'interposizione di una virgola (segno di regola non sprovvisto di valore), l'accento sintagmatico depone comunque a favore di un quam pressoché atono, a guisa di enclitica. Il passo successivo sarà la composizione, quando verrà eliminato lo spazio interposto tra la componente avverbio e la componente congiunzione di ante quam ${ }^{36}$.

Per il resto, varie ragioni ci hanno indotto ad anticipare la trattazione di uno o due altri accenti di frase: "Q(uod) reip(ublicae) causa non commoveri neq(ue) vellem, si possem, néq(ue) possem, si velim” (A4 $\left.{ }^{\mathrm{r}} 13-15\right)$, laddove nel primo inciso diversi sono gli elementi di risalto, mentre nell'ultimo inciso, in sede di lettura, va evidenziato e quasi scandito néque, il quale segna la transizione tra i due possem che si susseguono, uno in frase affermativa, l'altro in frase negativa.

Inoltre abbiamo annoverato tra i casi che si prestano a duplice interpretazione le due occorrenze di nónne $\left(\mathrm{C}^{\mathrm{V}} 1, \mathrm{C}^{\mathrm{V}} 16\right)$, in cui il segno può spiegarsi o come conseguenza di un uso estensivo dell'accento d'enclisi, oppure come indicatore dell'accento di frase, a segnare l'inizio del tono interrogativo che la connoterà.

${ }^{33}$ Un esempio di congiunzione subordinativa soggetta a diverso trattamento, in relazione all'accento di frase che si vuole imprimere, si ha ancora in Aldo Manuzio: "Differentia [sc. non servatur accentus], ut in quándo, quod interrogativu(m) acuit(ur), $\mathrm{cu}(\mathrm{m})$ aut $(\mathrm{em})$ significat ö $\tau$, gravatur, quandò": Manutius 1508, aa7v.

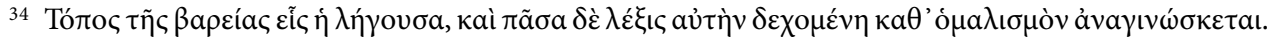

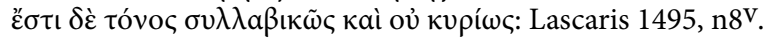

35 es corretto manualmente in esses nella copia fiorentina al nostro esame (correzione accolta nell'edizione del 1530, e pertanto sicuramente voluta dal Bembo).

${ }^{36}$ Nell'edizione Bembo 1530, molto meno ricca di accenti grafici, spariranno tale virgola e tale accento, mentre si manterrà in ante quam la divisione di parola. 


\section{Segni di quantità}

Ci siamo già soffermati sull'uso tonico del segno di accento circonflesso. Si registra poi un uso dello stesso segno con valore meramente diacritico, e non accentuale: mentre scriveva aliâs $\left(\mathrm{A} 2^{\mathrm{r}} 4\right)$, avverbio sul quale la lunga finale è contrassegnata con un segno identico a quello dell'accento circonflesso, forse nel Bembo operava il ricordo di quegli innumerevoli avverbi greci terminanti per - $\tilde{\omega} \varsigma$ che, essendo accentati sull'ultima, vi recano lo stesso circonflesso dell'aggettivo corrispondente in caso genitivo plurale.

In ultimo, in nobîs ("nobîs pueris", A7 ${ }^{\mathrm{V}} 2$ ) il segno del circonflesso, se, come sembra, vuol essere distintivo dell'ablativo dal dativo, si carica di un valore puramente diacritico, laddove l'un caso non si oppone all'altro per quantità ${ }^{37}$.

Il segno avrà una sua sopravvivenza nel tempo, fino ad edizioni dotte anche dell'Ottocento, e in sede scolastica almeno fino agli albori del Novecento.

\section{Apex inversus}

Vogliamo chiamar così un segno identico all'accento grave, adoperato per segnare l'ultima sillaba degli avverbi eo ed illo e di altri avverbi in -e. Deve trattarsi di un segno di quantità lunga, non orizzontale ma obliquo, visto che esso, almeno nella prassi accurata degli inizi, non insiste sugli avverbi in - $\breve{e}(\text { bene, male })^{38}$.

Considerato che ladozione di un segno coincidente con l'accento grave non era ovvia, non ci sottrarremo al dovere di avanzare un'ipotesi genetica. I Romani con tale funzione adoperavano l'apex, che, avendo la forma di un moderno accento acuto, era inclinato all'opposto: essendosi, all'interno della collaborazione Bembo-Manuzio, scelto di corredare il testo latino di un sistema di segni ispirato al greco, restava disponibile per l'ultima sillaba il detto segno coincidente con l'accento grave, grazie anche al fatto che questa non è sede di ictus se non nei casi di ossitonie secondarie ${ }^{39}$. Pertanto poté adottarsi l'espediente di invertire l'apex, applicando ad esso il trattamento che in greco si riserva all'acuto finale (di volgerlo in grave a patto che il discorso continui e non vi sia accento d'enclisi), sì da ricavarne un segno di lunga utilizzabile senza ambiguità ${ }^{40}$. Onde il nome di apex inversus da noi adottato.

${ }^{37}$ Impossibile dire se, a livello o consapevole o solamente psicologico, abbia giocato un ruolo ad es. il modello di i nuĩv. Tutta la flessione del pronome di prima e di seconda persona plurale in attico è notoriamente connotata da accento circonflesso sulle ultime sillabe ( $\pi \varepsilon \rho \iota \sigma \pi \omega ́ \mu \varepsilon v \alpha)$.

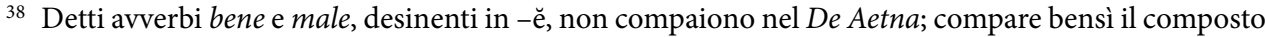
pérbene con un solo accento, l'acuto sulla sillaba iniziale. Stando così le cose, il controllo si può eseguire su altre Aldine latine di poco successive a questa: ad esempio negli Alexandri Benedicti Physici Diaria de bello Carolino pubblicati pochi mesi dopo, nellestate dello stesso 1496 (volumetto caratterizzato da netta propinquità formale nei confronti dell'opuscolo bembeo): da avverbi recanti il segno di lunga quali ferè (a $5^{r}$ $25,{ }^{2}{ }^{\mathrm{r}} 17, \mathrm{a} 7 \mathrm{~V} 5$ etc.) e penè $\left(\mathrm{a}^{\mathrm{V}} 19, \mathrm{~d} 2 \mathrm{~V}^{\mathrm{V}} 6, \mathrm{c}^{\mathrm{r}} 14\right.$ etc.) si distinguono nettamente bene $(\mathrm{a} 4 \mathrm{v} 22, \mathrm{c} 2 \mathrm{~V} 15, \mathrm{c} 7 \mathrm{~V} 5$, $\mathrm{g}^{\mathrm{V}} \mathrm{r}$ ) e male ( $\left.\mathrm{b} 4^{\mathrm{v}} 23, \mathrm{c} 8^{\mathrm{V}} 18, \mathrm{~d} 4 \mathrm{r}^{\mathrm{r}} 6, \mathrm{f}^{\mathrm{r}} 5, \mathrm{~g}^{\mathrm{r}} \mathrm{g}, \mathrm{g} 2^{\mathrm{V}} 1\right)$, i quali ne sono correttamente privi; e nelle Laurentii Maioli Genuensis Epiphyllídes in dialecticis (1497) si oppongono forme come ferè (c8v 12), $\approx$ feré ( $\left.\mathrm{g}^{\mathrm{V}} \mathrm{V} 31\right)$ a bene ( $3^{\mathrm{V}} 2832, \mathrm{c}^{\mathrm{r}} 7, \mathrm{f}^{\mathrm{v}} 16$ etc.), avverbio sempre privo dei segni omologhi.

${ }^{39}$ Che il latinista del Rinascimento era lungi dall'ignorare. Aldo Manuzio mostrerà di conoscere il tipo di "Arpinâs, Ravennâs, Cuiâs, Nostrâs" (che, come si vede, vuole contrassegnato da accento circonflesso, segno storicamente legato a fenomeni di contrazione) e diverse altre particolarità aa $6^{\mathrm{V}}$-aa $7 \mathrm{r}$ : Manutius 1508 , aa $8^{\mathrm{r}}$, aa6 ${ }^{\mathrm{V}}$-aa7r.

${ }^{40}$ Costantino Lascaris nella sua grammatica greca, gli Erotemata, si sofferma soltanto sul primo

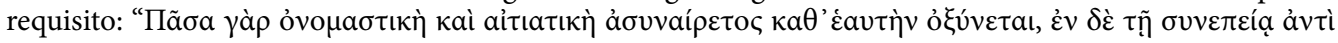


La frequenza del segno, qui esclusivo appannaggio di un esiguo numero di avverbi, è comunque abbastanza elevata, come mostra il breve elenco che segue:

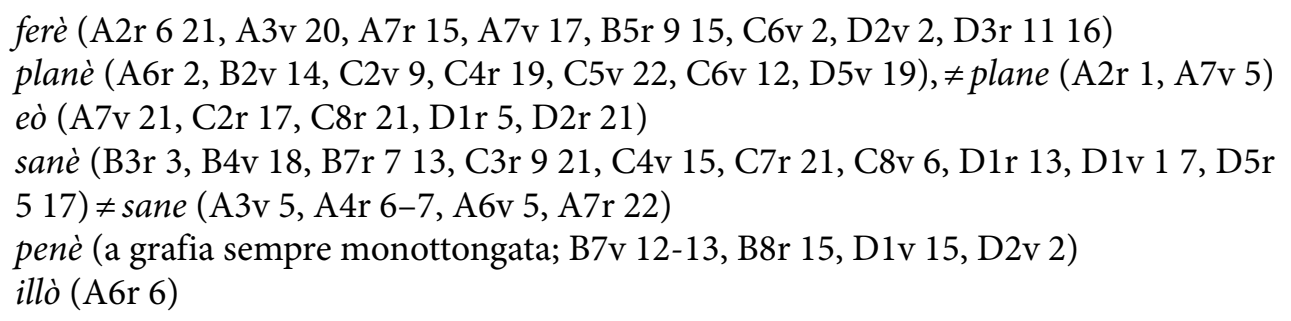

\section{Conclusioni}

Il corredo di accenti di cui Pietro Bembo provvide il De Aetna, fautore ed auspice Aldo Manuzio (ché un'operazione siffatta, piena di risvolti tecnici, non poteva compiersi senza la partecipazione e l'attivo sostegno della tipografia), rappresenta un sistema articolato e complesso, il quale, se appare orientato eminentemente al fine pratico di assicurare una lettura corretta ed espressiva del latino, fu comunque oggetto di un'attenta elaborazione teorica preliminare. Nella sperimentazione che avviarono non vè modo di distinguere il contributo personale di ciascuno, potendosi supporre che notevole sia stato anche l'apporto dell'umanista-stampatore, attivo anche come grammatico autore di apprezzati manuali, che di tale sperimentazione, negli anni, sarebbe stato oltretutto il convinto continuatore.

Determinante fu l'esperienza del greco, che culturalmente rappresentò il punto di partenza ed il referente rispetto al quale costruire il sistema di segni di cui si andava a corredare il latino. A porre le premesse teoriche remote delloperazione avevano provveduto quei grammatici latini (quali Servio e Prisciano) che, nel solco della grammatica tardorepubblicana e imperiale, ritennero trasferibile al latino la teoria dell'accento greco, inclusi i segni dell'accento grave e dell'accento circonflesso.

I tre segni di derivazione greca, grazie anche ad unaccorta polisemia sviluppata a partire da una sottile conoscenza della lingua fonte, bastarono a trasmettere, in un sistema che rimaneva sostanzialmente scevro di ambiguità, una comunicazione ricca di informazioni sulla corretta lettura del testo, dagli aspetti basilari fino a non poche sfumature espressive.

Diversi provvedimenti adottati in materia di accento sintagmatico e di accento di frase lasciano ritenere che il Bembo avesse posto mente non solo alla lettura privata, orale o mentale (endofasica) che fosse, ma anche ad uneventuale declamazione. La finezza di certe indicazioni, in particolare, fa pensare che esse siano state verificate in occasione di una lettura ad alta voce del testo, finalizzata alla ricerca di difficoltà che investissero non solo la singola parola, ma anche la stessa comprensione e lettura accurata della frase e del periodo.

Ladozione del greco a modello non comportò comunque l'apposizione di un accento grafico ad ogni sillaba tonica del testo, come tendenzialmente accadeva nelle edizioni

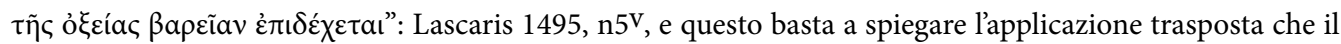
Bembo ne fa all'apex. D'altronde nessuna di quelle vocali lunghe che il Bembo connota con l'apex inversus è nel contempo sede di accento tonico. 
greche coeve, e accade tuttavia. L'accento d'enclisi, che risalta particolarmente per la sua sistematica apposizione nel testo, costituisce un caso a parte e dà adito all'interrogativo se esso abbia potuto rappresentare un ambito di incertezza, dottrinale o pratica, per il lettore medio in quella fase del Rinascimento italiano.

Per il resto, emerge il criterio di economia e di utilità seguito: si corredarono di accenti grafici tutti i termini ritenuti meritevoli di disambiguazione, nonché tutti quelli che potessero dare adito a incertezze di lettura, realizzando un documento simile a certi papiri greci di età romana, nei quali l'informazione sull'accento, sporadica, nel soccorrere il lettore laddove egli può trovarsi in difficoltà, risponde parimenti al criterio dell'utile.

Il testo che ci troviamo dinanzi consente di misurare di riflesso le conoscenze linguistiche di certo lettore parzialmente colto di età rinascimentale, ingenerando il sospetto che certe difficoltà prosodiche del lettore di oggi, comportanti errori ricorrenti di accentazione, esistano forse ab aevo.

\section{Bibliografia}

Bembo, P. Ad Angelum Chabrielem de Aetna liber. Impressum Venetiis, in aedibus Aldi Romani, mense Februario anno MVD (=1496). https://archive.org/details/ita-bnc-ald-00000039-001 (31.12.2015.)

Bembo, P. Ad Angelum Chabrielem de Aetna. Venetiis, per Io(annem) Ant(onium) eiusq(ue) Fratres de Sabio, 1530. https://archive.org/details/ita-bnc-mag-00000708-003 (31.12.2015.)

Benedictus, A. (Physicus). Diaria de bello Carolino. Impressum Venetiis, in aedibus Aldi Romani, 1496. http://issuu.com/bazzmann/docs/diaria-debello-carolino (31.12.2015).

Bühler, C. F. Manuscript corrections in the Aldine edition of Bembo's De Aetna. The Papers of the Bibliographical Society of America. 1951, 45/26 136-142.

Castellani, A. Sulla formazione del sistema paragrafematico moderno. Studi linguistici italiani. 1995, 21, 3-47.

Gualdo Rosa, L. Crastone, Giovanni, in: Dizionario Biografico degli Italiani. 1984, 30, 578-580.

Kühner, R. Ausführliche Grammatik der griechischen Sprache. 1. T. Elementar- und Formenlehre, bearbeitet von F. Blass. 1. B. 3. Aufl. Hannover, Hahn, 1890.

Lascaris, C. Grammatices Graecae epitome cum interpretatione Johannis monachi Placentini. Mediolani, curis Boni Accursii Pisani, 1480.

Lascaris, C. Erotemata $c u(m)$ interpretatione latina [...] Impressum Venetiis in aedibus Aldi Romani, 1495. https://archive.org/details/ita-bnc-ald-00000689-001 (31.12.2015.)

Loew, E. A. The Beneventan script, a History of the South Italian minuscule. $2^{\text {nd }}$ ed. by V.Brown. Roma, Edizioni di Storia e Letteratura, 1980.

Maioli, L. Genuensis. Epiphyllídes in dialecticis. Impressum Venetiis in aedibus Aldi Romani, 1497. https:// archive.org/details/ita-bnc-ald-00000691-001 (31.12.2015).

Manutius, A. Romanus. Institutionum grammaticarum libri quatuor. Venetiis apud Aldum, Aprili mense 1508. http://www.internetculturale.it/opencms/opencms/it/viewItemMag.jsp?case=\&id=oai\%3A193. 206.197.121\%3A18\%3AVE0049\%3AUM1E008629 (31.12.2015).

Mardersteig, G. Aldo Manuzio e i caratteri di Francesco Griffo da Bologna, in: id. Scritti sulla storia dei caratteri e della tipografia. Milano, Polifilo, 1988, 107-159.

Quicherat, L. Thesaurus poeticus linguce latino. Paris, Hachette, 1846.

Tinti, P. Griffo, Francesco, in: Dizionario Biografico degli Italiani 59, 2002, 377-380.

For citation: Di Maria G. Tra dottrina grammaticale e ortografia latina: l'Aldina del De Aetna di Pietro Bembo. Philologia Classica 2016, 11(1), 148-162. DOI: 10.21638/11701/spbu20.2016.113 
МЕЖДУ ГРАММАТИЧЕСКИМ УЧЕНИЕМ И ЛАТИНСКОЙ ОРФОГРАФИЕЙ: ТРАКТАТ ПЬЕТРО БЕМБО «ОБ ЭТНЕ» В ИЗДАНИИ АЛЬДА МАНУЦИЯ

Джорджо ди Мария

Диакритические знаки, которыми Пьетро Бембо с помощью Альда Мануция снабдил свой диалог «Об Этне», представляют собой четкую и сложную систему. И хотя эта система была ориентирована на обеспечение правильного и выразительного чтения латинского текста, то есть ставила перед собой практическую цель, однако для ее создания требовалась тщательная теоретическая разработка. Решающую роль сыграл опыт работы с текстами на греческом языке. Он стал как отправной точкой, так и основой для построения системы знаков. Нельзя не отметить также влияние латинских грамматиков, таких как Сервий и Присциан, которые считали возможным применение к латинскому языку теории греческих ударений. Трех надстрочных знаков хватило для того, чтобы дать четкое представление не только об основах правильного чтения, но и о многочисленных нюансах. Несколько решений, принятых автором относительно синтагменного и фразового ударений, позволяют нам предположить, что Бембо предусматривал как приватное чтение, так и публичное выступление.

Ключевые слова: Пьетро Бембо, Альд Мануций, трактат «Об Этне», акцентуация.

Received: 08.01.2016

Final version received: 29.04.2016 